\title{
An Uncertainty Principle for Real Signals in the Fractional Fourier Transform Domain
}

\author{
Sudarshan Shinde and Vikram M. Gadre
}

\begin{abstract}
The fractional Fourier transform (FrFT) can be thought of as a generalization of the Fourier transform to rotate a signal representation by an arbitrary angle $\alpha$ in the time-frequency plane. A lower bound on the uncertainty product of signal representations in two FrFT domains for real signals is obtained, and it is shown that a Gaussian signal achieves the lower bound. The effect of shifting and scaling the signal on the uncertainty relation is discussed. An example is given in which the uncertainty relation for a real signal is obtained, and it is shown that this relation matches with that given by the uncertainty relation derived.
\end{abstract}

Index Terms-Fractional Fourier transform, time-frequency analysis, uncertainty principle.

\section{INTRODUCTION}

$\mathbf{T}$ HE uncertainty principle in the time-frequency plane plays an important role in signal processing. This principle states that for a given unit energy signal $x(t)$ with Fourier transform $X(j \omega)$, the product of spreads of the signal in time domain and frequency domain is bounded by a lower bound

$$
\int_{-\infty}^{\infty}\left|\left(t-t_{0}\right) x(t)\right|^{2} d t \int_{-\infty}^{\infty}\left|\left(\omega-\omega_{0}\right) X(j \omega)\right|^{2} d \omega \geq \frac{1}{4}
$$

where

$$
\begin{aligned}
t_{0} & =\int_{-\infty}^{\infty} t|x(t)|^{2} d t \\
\omega_{0} & =\int_{-\infty}^{\infty} \omega|X(\omega)|^{2} d \omega .
\end{aligned}
$$

The fractional Fourier transform (FrFT) can be thought of as a generalization of the Fourier transform, in which the signal

Manuscript received February 12, 2001; revised July 17, 2001. This work was supported by the Naval Research Board of India in the project entitled "Transient Detection using Wavelets." The associate editor coordinating the review of this paper and approving it for publication was Dr. Olivier Cappe.

The authors are with the Department of Electrical Engineering, Indian Institute of Technology, Bombay, India (e-mail: shinde@ee.iitb.ac.in; vmgadre@ee.iitb.ac.in).

Publisher Item Identifier S 1053-587X(01)09217-0. representation can be thought of as getting rotated by an angle $\alpha$ in the time-frequency plane. It is natural to ask the following: What kind of uncertainty relation will the time and FrFT spreads obey?

It has already been shown [1], [2] that if $X\left(u_{\alpha}\right)$ is the FrFT of $x(t)$, then a lower bound on the product of time and FrFT spreads is given by $\left(\sin ^{2} \alpha / 4\right)$. This lower bound can also be obtained by using the relationship between Fourier transform and FrFT given in [3] and uncertainty relation in the time-frequency domain. Uncertainty relations in the FrFT domain are also discussed in [4] and [5]. In [4], it is shown that the product of time spread and frequency spread of a signal are not invariant under FrFT. Many other measures that are invariant under FrFT are obtained in this paper. However, this paper does not talk about the product of the spreads of a signal in time and FrFT domains.

In this paper, we achieve a lower bound on the product of the spreads in time and FrFT domain for real $x(t)$. This lower bound is a tighter lower bound than that given in [1] on the uncertainty product of signal representations in two FrFT domains. This lower bound can be achieved by a Gaussian signal.

\section{Fractional Fourier TRANSFORM}

The FrFT operator $\mathbf{R}_{\alpha}$, which rotates the signal representation by an angle $\alpha$ in the time-frequency plane, is defined as [6]

$$
X\left(u_{\alpha}\right) \stackrel{\text { def }}{=}\left(\mathbf{R}_{\alpha} x\right)\left(u_{\alpha}\right) \stackrel{\text { def }}{=} \int_{-\infty}^{\infty} x(t) K_{\alpha}\left(t, u_{\alpha}\right) d t
$$

where we have (5), shown at the bottom of the page.

The inverse FrFT is given by

$$
x(t)=\int_{-\infty}^{\infty} X\left(u_{\alpha}\right) K_{\alpha}^{*}\left(u_{\alpha}, t\right) d u_{\alpha} .
$$

Note that FrFT reduces to the ordinary Fourier transform for $\alpha=\pi / 2$.

The following properties of $K_{\alpha}(u, t)$, given in [6] and [7], will be useful later.

$$
\begin{array}{r}
K_{\alpha}\left(t, u_{\alpha}\right)=K_{\alpha}\left(u_{\alpha}, t\right) \\
K_{-\alpha}\left(t, u_{\alpha}\right)=K_{\alpha}^{*}\left(t, u_{\alpha}\right)
\end{array}
$$

$$
K_{\alpha}\left(t, u_{\alpha}\right)= \begin{cases}\sqrt{\frac{1-j \cot \alpha}{2 \pi}} \exp \left(j \frac{t^{2}+u_{\alpha}^{2}}{2} \cot \alpha-j u_{\alpha} t \csc \alpha\right), & \text { if } \alpha \text { not a multiple of } \pi \\ \delta\left(t-u_{\alpha}\right), & \text { if } \alpha \text { a multiple of } 2 \pi \\ \delta\left(t+u_{\alpha}\right), & \text { if } \alpha+\pi \text { a multiple of } 2 \pi\end{cases}
$$




$$
\begin{aligned}
\int_{-\infty}^{\infty}|x(t)|^{2} d t= & \int_{-\infty}^{\infty}\left|X\left(u_{\alpha}\right)\right|^{2} d u_{\alpha} \\
\int_{-\infty}^{\infty} t x(t) K_{\alpha}\left(u_{\alpha}, t\right) d t= & \cos (\alpha) u_{\alpha} X\left(u_{\alpha}\right) \\
& +j \sin (\alpha) \frac{d}{d u_{\alpha}} X\left(u_{\alpha}\right)
\end{aligned}
$$

\section{UNCERTAINTY PRINCIPLE IN FrFT DOMAIN}

Let $x(t)$ be a unit energy real-valued signal such that $t x(t) \in$ $L_{2}(R)$. We define the following:

$$
\begin{aligned}
\mathbf{T} x(t) & \stackrel{\text { def }}{=} t x(t) \\
\mathbf{W} x(t) & \stackrel{\text { def }}{=}-j \frac{d}{d t} x(t) \\
\mathbf{L}_{\alpha} & \stackrel{\text { def }}{=} \cos (\alpha) \mathbf{T}-\sin (\alpha) \mathbf{W} \\
t_{0} & \stackrel{\text { def }}{=} \int_{-\infty}^{\infty} t|x(t)|^{2} d t \\
u_{\alpha 0} & \stackrel{\text { def }}{=} \int_{-\infty}^{\infty} u_{\alpha}\left|X\left(u_{\alpha}\right)\right|^{2} d u_{\alpha} \\
\Delta t^{2} & \stackrel{\text { def }}{=} \int_{-\infty}^{\infty}\left|\left(t-t_{0}\right) x(t)\right|^{2} d t \\
\Delta u_{\alpha}{ }^{2} & \stackrel{\text { def }}{=} \int_{-\infty}^{\infty}\left|\left(u_{\alpha}-u_{\alpha 0}\right) X\left(u_{\alpha}\right)\right|^{2} d u_{\alpha} .
\end{aligned}
$$

Then

$$
\begin{aligned}
\Delta t^{2}+t_{0}^{2} & =\int_{-\infty}^{\infty}|\mathbf{T} x(t)|^{2} d t \\
\Delta u_{\alpha}{ }^{2}+u_{\alpha 0}^{2} & =\int_{-\infty}^{\infty}\left|\mathbf{L}_{\alpha}^{*} x(t)\right|^{2} d t
\end{aligned}
$$

where (19) follows from the properties of $K_{\alpha}\left(u_{\alpha}, t\right)$ in (7)-(10).

Lemma 1: If $x(t)$ is real with $t_{0}=0$, then $u_{\alpha 0}=0$.

Proof: Interchanging the role of time and FrFT domains in (10), it follows that the inverse FrFT of $u_{\alpha} X\left(u_{\alpha}\right)$

$$
\begin{aligned}
\int_{-\infty}^{\infty} u_{\alpha} X\left(u_{\alpha}\right) K_{\alpha}^{*}\left(u_{\alpha}, t\right) d u_{\alpha} \\
=\cos (\alpha) t x(t)-j \sin (\alpha) \frac{d x(t)}{d t} .
\end{aligned}
$$

It is known [6] that

$$
\int_{-\infty}^{\infty} Y\left(u_{\alpha}\right) X^{*}\left(u_{\alpha}\right) d u_{\alpha}=\int_{-\infty}^{\infty} y(t) x^{*}(t) d t .
$$

Choosing $Y\left(u_{\alpha}\right)=u_{\alpha} X\left(u_{\alpha}\right)$ and noting that $x(t)$ is real

$$
\begin{aligned}
& \int_{-\infty}^{\infty} u_{\alpha} X\left(u_{\alpha}\right) X^{*}\left(u_{\alpha}\right) d u_{\alpha} \\
& \quad=\int_{-\infty}^{\infty}\left(\cos (\alpha) t x(t)-j \sin (\alpha) \frac{d x(t)}{d t}\right) x(t) d t .
\end{aligned}
$$

Using the facts that $x(t)$ is real, $t_{0}=0$, and $\lim _{t \rightarrow \pm \infty} x^{2}(t)=0$ since $x(t) \in L_{2}(R)$, we get that the right-hand side of (23) is equal to zero. Since the left-hand side is equal to $u_{\alpha 0}$, we have $u_{\alpha 0}=0$, and the lemma is proved.
Theorem 1 (Uncertainty Principle in the FrFT Domain): If $x(t)$ is a unit energy real-valued signal, and $0 \leq \alpha, \beta \leq \pi / 2$, then

$$
\begin{gathered}
\Delta u_{\alpha}{ }^{2} \Delta u_{\beta}{ }^{2} \geq\left[\Delta t^{2} \cos \alpha \cos \beta+\frac{\sin \alpha \sin \beta}{4 \Delta t^{2}}\right]^{2} \\
+\frac{\sin ^{2}(\alpha-\beta)}{4}
\end{gathered}
$$

and equality is achieved when

$$
x(t)=\left(\frac{1}{\pi \sigma^{2}}\right)^{\frac{1}{4}} \exp \left(-\frac{t^{2}}{2 \sigma^{2}}\right)
$$

where $\sigma$ is an arbitrary real constant.

Proof: Without loss of generality, we take $t_{0}=0$. If $t_{0}$ is not equal to zero, we can shift the signal appropriately to make it zero. It will be shown in the next section that shifting the signal does not affect the uncertainty relation. Since $t_{0}=0$, by Lemma 1 for any angle $\alpha$, we have $u_{\alpha 0}=0$, and from (19)

$$
\Delta u_{\alpha}^{2}=\int_{-\infty}^{\infty}\left|\mathbf{L}_{\alpha}^{*} x(t)\right|^{2} d t .
$$

From the Cauchy-Schwartz inequality

$$
\begin{aligned}
\left|\int_{-\infty}^{\infty} \mathbf{L}_{\alpha}^{*} x(t)\left(\mathbf{L}_{\beta}^{*} x\right)^{*}(t) d t\right|^{2} & \leq \int_{-\infty}^{\infty}\left|\mathbf{L}_{\alpha}^{*} x(t)\right|^{2} d t \\
& \times \int_{-\infty}^{\infty}\left|\mathbf{L}_{\beta}^{*} x(t)\right|^{2} d t
\end{aligned}
$$

Now, for real $x(t)$

$$
\begin{aligned}
\left|\int_{-\infty}^{\infty} \mathbf{L}_{\alpha}^{*} x(t)\left(\mathbf{L}_{\beta}^{*} x\right)^{*}(t) d t\right|^{2} \\
=\mid \int_{-\infty}^{\infty}(\mathbf{T} \cos \alpha+\mathbf{W} \sin \alpha) x(t) \\
\quad \times\left.(\mathbf{T} \cos \beta-\mathbf{W} \sin \beta) x(t) d t\right|^{2} .
\end{aligned}
$$

Expanding the right-hand side and noting that by putting $\alpha=$ $\pi / 2$ in (26), we have

$$
\int_{-\infty}^{\infty}|\mathbf{W} x(t)|^{2} d t=\Delta u_{\pi / 2}^{2}
$$

and for a real-valued function $x(t)$ such that $t x(t) \in L_{2}(R)$

$$
\int_{-\infty}^{\infty} \mathbf{T} x(t) \mathbf{W} x(t) d t=-\frac{j}{2} \text {. }
$$

Therefore

$$
\begin{array}{r}
\left|\int_{-\infty}^{\infty} \mathbf{L}_{\alpha}^{*} x(t)\left(\mathbf{L}_{\beta}^{*} x\right)^{*}(t) d t\right|^{2}=\left[\Delta t^{2} \cos \alpha \cos \beta\right. \\
\left.+\Delta u_{\pi / 2}^{2} \sin \alpha \sin \beta\right]^{2}+\frac{\sin ^{2}(\alpha-\beta)}{4} .
\end{array}
$$

Since $u_{\pi / 2}$ is a frequency-domain variable, from the uncertainty relation between time and frequency

$$
\Delta u_{\pi / 2}^{2} \geq \frac{1}{4 \Delta t^{2}}
$$


From (26), (27), (31), and (32)

$$
\begin{gathered}
\Delta u_{\alpha}^{2} \Delta u_{\beta}^{2} \geq\left[\Delta t^{2} \cos \alpha \cos \beta+\frac{\sin \alpha \sin \beta}{4 \Delta t^{2}}\right]^{2} \\
+\frac{\sin ^{2}(\alpha-\beta)}{4}
\end{gathered}
$$

This inequality turns to equality only if there exists a function for which inequalities (27) and (32) become equalities. The Cauchy-Schwartz inequality (27) becomes an equality if for an arbitrary constant $c, x(t)$ satisfies

$$
\mathbf{L}_{\alpha}^{*} x(t)=c \mathbf{L}_{\beta}^{*} x(t)
$$

Solving this differential equation, we get

$$
x(t)=k \exp \left(\frac{-j(\cos \alpha-\cos \beta)}{\sin \alpha-c \sin \beta} \frac{t^{2}}{2}\right)
$$

where $k$ is a constant of integration. In order to make $x(t)$ real valued, $c$ can be chosen as

$$
c=\frac{\sigma^{2} \cos \alpha+j \sin \alpha}{\sigma^{2} \cos \beta+j \sin \beta}
$$

where $\sigma$ is an arbitrary constant. The value of $k$ can be found out by noting that $x(t)$ must be unit norm.

Thus, $x(t)$, which satisfies the Cauchy-Schwartz inequality (27), turns out to be a Gaussian function. It is well known that the Gaussian function also turns uncertainty inequality (32) to an equality. Thus, the Gaussian function given by (25) indeed turns inequality (24) into an equality, and the theorem is proved.

\section{EFFECT OF SHIFT AND ScALING}

It is known that in the case of the conventional Fourier Transform, the product of time and frequency spreads does not change with a shift or a scaling of the signal, and the lower limit on this product given by the uncertainty principle remains the same with shift or scaling. In this section we study the effect of shifting and scaling the signal on the uncertainty relation in time-FrFT domain.

Given a signal $x(t)$, it is known [6] that FrFT of the shifted signal $y(t)=x(t-\tau)$ is

$$
\begin{aligned}
Y\left(u_{\alpha}\right)= & X\left(u_{\alpha}-\tau \cos \alpha\right) \\
& \times \exp \left(j \frac{\tau^{2}}{2} \sin \alpha \cos \alpha-j u_{\alpha} \tau \sin \alpha\right) .
\end{aligned}
$$

Thus, spread of the signal changes in neither the time nor the FrFT domain; therefore, shifting the signal has no effect on the uncertainty relation.

Coming to scaling, let $y(t)=c^{1 / 2} x(c t)$. We represent time uncertainty of $x(t)$ by $\Delta t_{x}$ and the uncertainty in the FrFT domain at an angle $\alpha$ by $\Delta u_{x \alpha}$, and we use similar notations for $y(t)$. It is known that [6]

$$
Y\left(u_{\alpha}\right)=e^{j \chi_{\alpha}}\left(\frac{\sin \theta}{c \sin \alpha}\right)^{1 / 2} X\left(u_{\theta} \frac{\sin \theta}{c \sin \alpha}\right)
$$

where

$$
\tan \theta=c^{2} \tan \alpha
$$

and some phase factor $\chi_{\alpha}$ that is not of much importance here. Similarly, we can write

$$
Y\left(u_{\beta}\right)=e^{j \chi_{\beta}}\left(\frac{\sin \xi}{c \sin \beta}\right)^{1 / 2} X\left(u_{\xi} \frac{\sin \xi}{c \sin \beta}\right)
$$

where

$$
\tan \xi=c^{2} \tan \beta
$$

It can be shown that

$$
\begin{aligned}
\Delta t_{y}^{2} & =\frac{1}{c^{2}} \Delta t_{x}^{2} \\
\Delta u_{y \alpha}^{2} & =\frac{c^{2} \sin ^{2} \alpha}{\sin ^{2} \theta} \Delta u_{x \theta}^{2} \\
\Delta u_{y \beta}^{2} & =\frac{c^{2} \sin ^{2} \beta}{\sin ^{2} \xi} \Delta u_{x \xi}^{2}
\end{aligned}
$$

and we get

$$
\Delta u_{y \alpha}^{2} \Delta u_{y \beta}^{2}=\frac{c^{4} \sin ^{2} \alpha \sin ^{2} \beta}{\sin ^{2} \theta \sin ^{2} \xi} \Delta u_{x \theta}^{2} \Delta u_{x \xi}^{2} .
$$

If the lower limit on $\Delta u_{y \alpha}^{2} \Delta u_{y \beta}^{2}$, which is given by the right-hand side of inequality (24), is denoted by $L\left(\Delta t_{y}, \alpha, \beta\right)$, and the lower limit on $\Delta u_{x \theta}^{2} \Delta u_{x \xi}^{2}$ is denoted by $L\left(\Delta t_{x}, \theta, \xi\right)$, then it can be shown that

$$
L\left(\Delta t_{y}, \alpha, \beta\right)=\frac{c^{4} \sin ^{2} \alpha \sin ^{2} \beta}{\sin ^{2} \theta \sin ^{2} \xi} L\left(\Delta t_{x}, \theta, \xi\right) .
$$

Thus, scaling changes the product of spreads of the signal, and it changes the lower limits on these products by a proportional amount. It follows from (44) and (45) that if an uncertainty relation for a signal $x(t)$ is given, that is

$$
\Delta u_{x \theta}^{2} \Delta u_{x \xi}^{2} \geq L\left(\Delta t_{x}, \theta, \xi\right)
$$

and then multiplying both the sides of it by $\left(c^{4} \sin ^{2} \alpha \sin ^{2} \beta / \sin ^{2} \theta \sin ^{2} \xi\right.$ ), we can get an uncertainty relation for $y(t)$, which is the scaled version of the signal.

It can be seen that the uncertainty relation derived here is not robust to scaling since the lower limit depends on spread of the signal in the time domain. The major drawback of this is that given the signal spread in one domain, the lower limit on the spread in the other domain cannot be found out without the knowledge of $\Delta t^{2}$. One way to get around this problem is to replace the term containing the spread of the signal in the lower limit by its lowest value over all $\Delta t^{2}$. Since $\Delta t^{2} \in R^{+}$, it can be shown that

$$
\begin{aligned}
\min _{\Delta t^{2} \in R^{+}}\left[\Delta t^{2} \cos \alpha \cos \beta\right. & \left.+\frac{\sin \alpha \sin \beta}{4 \Delta t^{2}}\right]^{2} \\
& =\frac{1}{4} \sin 2 \alpha \sin 2 \beta
\end{aligned}
$$

and the uncertainty relation can be written as

$$
\Delta u_{\alpha}{ }^{2} \Delta u_{\beta}{ }^{2} \geq \frac{1}{4} \sin 2 \alpha \sin 2 \beta+\frac{\sin ^{2}(\alpha-\beta)}{4} .
$$

Remark: If $\alpha=\beta$ in (48), then it can be seen that $\Delta u_{\alpha}^{2} \geq$ $(\sin 2 \alpha / 2)$, proving that if the signal is real, its spread in the 
FrFT domain has a lower limit, irrespective of its spread in the time domain.

Note that (48) does not give any advantage for $\alpha=0$, but it can be seen that for $\alpha=0$, the uncertainty relation (24) reduces to

$$
\Delta u_{\beta}^{2} \Delta t^{2} \geq\left(\Delta t^{2}\right)^{2} \cos ^{2} \beta+\frac{\sin ^{2} \beta}{4} .
$$

This relation is only between two spreads, and even though it does not give the lower bound on the product of the spreads independent of $\Delta t^{2}$, since the uncertainty relation has reduced to a relation between only two spreads, given one spread, the lower limit on the other spread can be calculated without the knowledge of the spread in any third domain.

\section{EXAMPLE}

Let $x(t)=(2 / \sqrt{\pi})^{1 / 2} t \exp \left(-\left(t^{2} / 2\right)\right)$. Since this function is of the form $x(t)=k H_{1}(t) \exp \left(-\left(t^{2} / 2\right)\right)$, where $H_{1}(t)$ is the Hermite polynomial of degree 1 and $k$ is chosen to make $x(t)$ unit norm, it is known [7] that $x(t)$ is an eigenfunction of the FrFT operator, that is

$$
\mathbf{R}_{\alpha}[x(t)]=-\exp (j \alpha)\left(\frac{2}{\sqrt{\pi}}\right)^{1 / 2} t \exp \left(-\frac{u_{\alpha}^{2}}{2}\right)
$$

It can be easily calculated that $\Delta t^{2}=(3 / 2)$. It can also be seen that $\Delta t^{2}=\Delta u_{\alpha}^{2}$ for all $\alpha$. Then

$$
\begin{aligned}
& {\left[\Delta t^{2} \cos \alpha \cos \beta+\frac{\sin \alpha \sin \beta}{4 \Delta t^{2}}\right]^{2}+\frac{\sin ^{2}(\alpha-\beta)}{4}} \\
& =\frac{9}{4}\left[\frac{1}{81}+\frac{8 \cos ^{2} \alpha}{81}+\frac{8 \cos ^{2} \beta}{81}+\frac{64 \cos ^{2} \alpha \cos ^{2} \beta}{81}\right] \\
& \leq \frac{9}{4} \\
& =\Delta u_{\alpha}^{2} \Delta u_{\beta}^{2} \text {. }
\end{aligned}
$$

Thus, $\Delta u_{\beta}^{2} \Delta u_{\alpha}^{2}$ is indeed greater than the limit defined on it by the uncertainty theorem.

Consider now $y(t)=\sqrt{c} x(c t)$. It can be shown that

$$
\begin{aligned}
& \mathbf{R}_{\alpha}[y(t)]=\sqrt{\frac{c(1-j \cot \alpha)}{c^{2}-j \cot \alpha}} \exp (j(\delta-\alpha)) x \\
& \times\left(u \frac{\sin \theta}{c \sin \alpha}\right)
\end{aligned}
$$

where $\tan \theta=c^{2} \tan \alpha$. It can be seen that for $0<\alpha<\pi / 2$, $\Delta t_{y}^{2}=\left(c^{2} \sin ^{2} \alpha / \sin ^{2} \theta\right) \Delta t_{x}^{2}=\left(c^{2} \sin ^{2} \alpha / \sin ^{2} \theta\right)(3 / 2)=$ $(3 / \sin 2 \theta)(\sin 2 \alpha / 2) \geq(\sin 2 \alpha / 2)$ as asserted by the remark given in the previous section.

\section{CONCLUSION}

In this paper, we achieved a tighter lower bound than that is given in [1] on the product of uncertainties in two FrFT domains for real signals. It is also shown that this lower bound is achieved by a real-valued Gaussian signal. It is also shown that shifting does not affect the uncertainty relation, and an uncertainty relation for a scaled version of a signal can be derived by multiplying an uncertainty relation for the signal by an appropriate factor.

Future work in this direction involves finding out how these results can be generalized to complex signals and how the uncertainty principle can be extended to the discrete domain.

\section{REFERENCES}

[1] H. M. Ozaktas and O. Aytur, "Fractional fourier domains," Signal Process., vol. 46, pp. 119-124, Sept. 1995.

[2] O. Aytur and H. M. Ozaktas, "Non-orthogonal domains in phase space of quantum optics and their relation to fractional Fourier transform," Opt. Commun., vol. 120, pp. 166-170, 1995.

[3] A. I. Zayed, "On the relationship between fourier and fractional Fourier transforms," IEEE Signal Processing Lett., vol. 3, pp. 310-311, Dec. 1996.

[4] D. Mustard, "Uncertainty principle invariant under fractional Fourier transform,” J. Austral. Math. Soc. Ser. B, vol. 33, pp. 180-191, 1991.

[5] H. M. Ozaktas, Z. Zalevsky, and M. A. Kutay, The Fractional Fourier Transform with Applications in Optics and Signal Processing. New York: Wiley, 2000.

[6] A. L. Almeida, "The fractional Fourier transform and time-frequency representations," IEEE Trans. Signal Processing, vol. 42, pp. 3084-3091, Nov. 1994

[7] A. C. McBride and F. H. Kerr, "On Namias' fractional Fourier transform,” IMA J. Appl. Math., vol. 39, pp. 159-175, 1987.

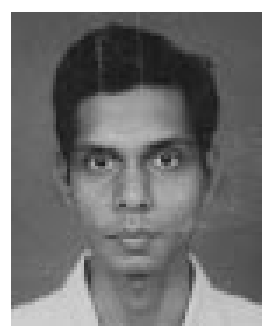

Sudarshan Shinde received the B.E. degree from G.S.I.T.S. Indore, India, in 1991 and the M.Tech. degree from the Indian Institute of Technology (IIT), Madras, in 1994. He has been pursuing the the Ph.D. degree at the Indian Institute of Technology (IIT) Bombay, Mumbai, since July 1997.

From 1994 to 1997, he was a software engineer with Infosys Technologies, Ltd., Bangalore, India. He has been supported by a fellowship from the Naval Research Board of India during his Ph.D. studies.

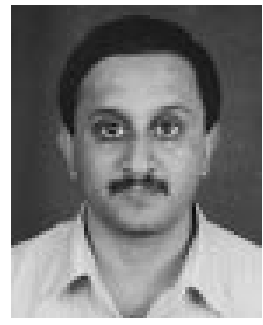

Vikram M. Gadre received the B.Tech. degree in electrical engineering from the Indian Institute of Technology (IIT), Delhi, in 1989 and the Ph.D. degree from IIT in 1994.

He is currently with the faculty of the Department of Electrical Engineering, Indian Institute of Technology (IIT) Bombay, Mumbai, India. His research interests lie broadly in communication and signal processing, particularly in multiresolution approaches.

Dr. Gadre received the President of India Gold Medal from IIT Delhi in 1989 and the Award for Excellence in Teaching from IIT Bombay in 1999. 